\title{
Multifunctional Inorganic Nanoparticles for Imaging, Targeting, and Drug Delivery
}

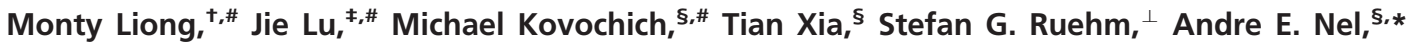 \\ Fuyuhiko Tamanoi, ${ }^{ \pm, *}$ and Jeffrey I. Zink ${ }^{\dagger, *}$ \\ ${ }^{\dagger}$ Department of Chemistry and Biochemistry, ${ }^{\ddagger}$ Department of Microbiology, Immunology, and Molecular Genetics, ${ }^{5}$ Department of Medicine, and ${ }^{\perp}$ Department of \\ Radiological Sciences, University of California, Los Angeles, California, 90095. "These authors contributed equally to this work.
}

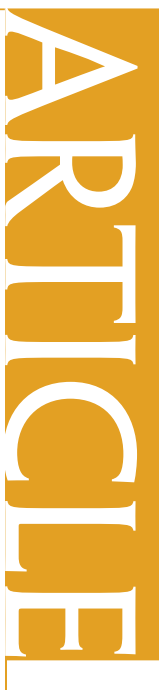

$\mathrm{P}$

rogress in utilizing inorganic nanoparticles for biomedical applications has advanced rapidly due to the extensive amount of work done in the synthesis and modification of the materials. ${ }^{1-6}$ These nanosized materials provide a robust framework in which two or more components can be incorporated to give multifunctional capabilities. An example can be seen in gold nanomaterials: the ability to control the size and shape of the particles and their surface conjugation with antibodies allow for both selective imaging and photothermal killing of cancer cells by using light with longer wavelengths for tissue penetration. ${ }^{7-9}$ Similar success was also demonstrated with polymer-coated superparamagnetic iron oxide nanoparticles. By conjugating multiple components such as fluorescent molecules, tumor-targeting moieties, anticancer drugs, or siRNA to the polymeric coating, not only can these multifunctional nanoparticles target human cancers, they can also be imaged inside the body by both magnetic resonance (MR) and fluorescence imaging. ${ }^{10,11}$ The capabil-
ABSTRACT Drug delivery, magnetic resonance and fluorescence imaging, magnetic manipulation, and cell targeting are simultaneously possible using a multifunctional mesoporous silica nanoparticle. Superparamagnetic iron oxide nanocrystals were encapsulated inside mesostructured silica spheres that were labeled with fluorescent dye molecules and coated with hydrophilic groups to prevent aggregation. Water-insoluble anticancer drugs were delivered into human cancer cells; surface conjugation with cancer-specific targeting agents increased the uptake into cancer cells relative to that in non-cancerous fibroblasts. The highly versatile multifunctional nanoparticles could potentially be used for simultaneous imaging and therapeutic applications.

KEYWORDS: mesoporous silica $\cdot$ iron oxide $\cdot$ dual imaging $\cdot$ drug delivery $\cdot$ multifunctional

ity to simultaneously image and treat tumors with nanoparticles may prove advantageous over conventional chemotherapy.

In this text, we describe the synthesis of multifunctional inorganic nanoparticles that are designed for cancer cell-specific delivery of hydrophobic anticancer drugs and have dual-imaging capability (optical and MR) (Figure 1). Superparamagnetic iron oxide nanocrystals ( $20 \mathrm{~nm}$ ) were incorporated in the mesoporous silica nanoparticles (100-200 nm) for the magnetic manipulation and MR imaging. Surface

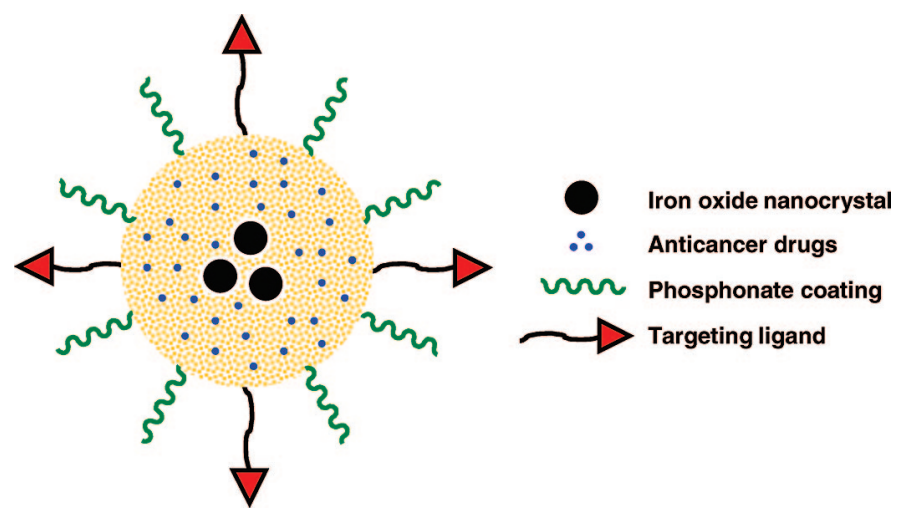

Figure 1. Schematic illustration of multifunctional nanoparticles showing iron oxide nanocrystals encapsulated within mesoporous silica, hydrophobic anticancer drugs stored inside the pores, and surface modifications with phosphonate and folic acid targeting ligands. 


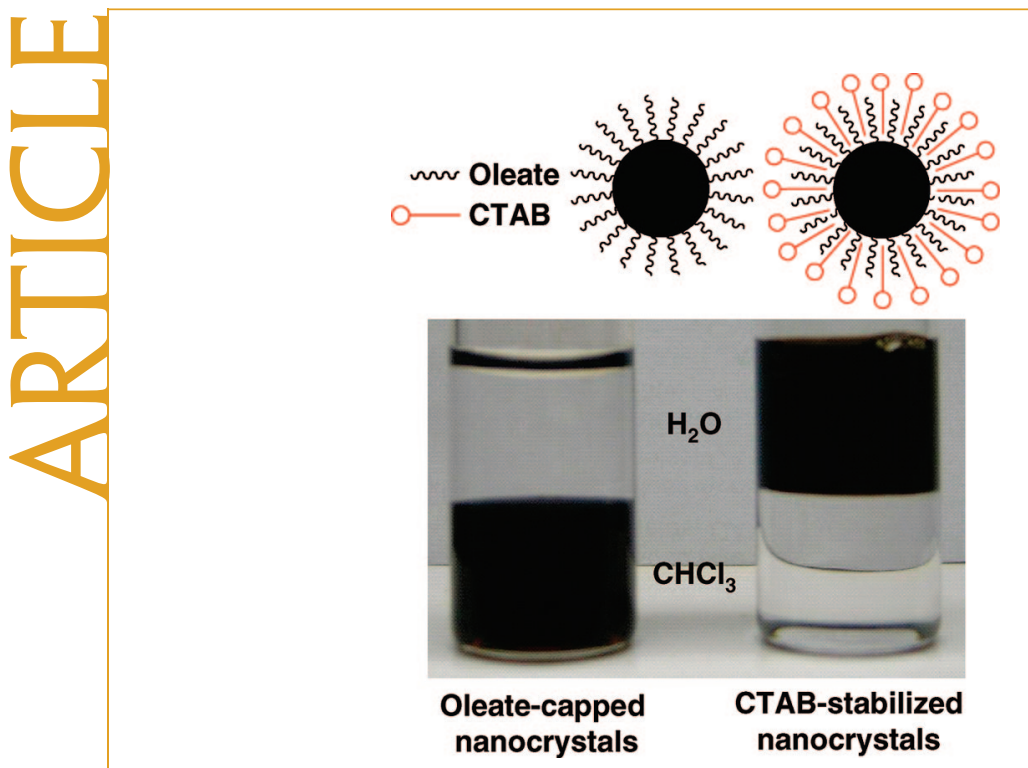

Figure 2. (Left) As-synthesized oleate-capped iron oxide nanocrystals in chloroform and (right) water-soluble CTABstabilized nanocrystals.

attachment with hydrophilic groups increased the stability of the nanoparticle dispersion in aqueous solution. The mesoporous silicate was further modified with fluorescent molecules and targeting ligands, and the pores were filled with chemotherapeutic drug molecules.

We demonstrate that these nanoparticles can be monitored inside living cells by both MR and fluorescence imaging methods and simultaneously used as a drug delivery vehicle. The targeting ligand modification increased the drug payload delivery into human cancer cells relative to that into non-cancerous cells. The synthetic procedures require inexpensive and nonhazardous precursors and are simple enough for largescale production. The potential to simultaneously monitor and deliver molecules to the targeted tissue region will be highly beneficial for both imaging and therapeutic purposes.

\section{RESULTS AND DISCUSSION}

Magnetic Functionality. Superparamagnetic iron oxide nanocrystals were used as the functional component that can provide the MR imaging and magnetic manipulation capabilities. We followed the hightemperature and non-aqueous route to synthesize the
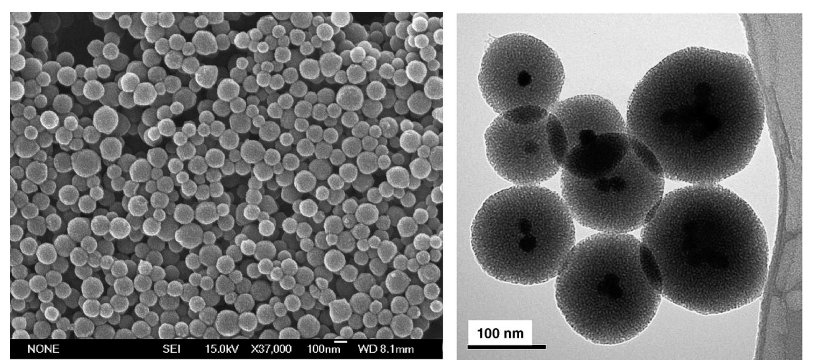

Figure 3. (Left) Scanning electron microscope and (right) transmission electron microscope images of the iron oxide incorporated within the mesoporous silica NPs. magnetic nanocrystals in order to produce highly uniform and crystalline particles. ${ }^{12-16}$ The nanocrystals were synthesized by the thermal decomposition of iron-oleate complexes in a solution of oleic acid surfactants and octadecene solvent because this facile procedure utilizes inexpensive reagents and yields large quantities of the materials (Figure S-1, Supporting Information). ${ }^{13}$ The hydrophobic nanocrystals dissolved in chloroform were transferred to the water phase by mixing them with an aqueous cetyltrimethylammonium bromide (CTAB) solution and evaporating the organic solvent. ${ }^{17-19}$ Using this simple method, the hydrophobic tail of the CTAB surfactant interacts strongly with the hydrophobic oleate ligand on the surface of the nanocrystals, and the hydrophilic charged headgroup of CTAB makes the nanocrystals water-soluble. The transfer of the nanocrystals into aqueous solution was highly effective since there were no visible precipitates or aggregates in the solution (Figure 2).

Mesoporous Silica Formation. Silica offers many advantages as the framework for the multifunctional nanoparticle. In addition to being able to incorporate other inorganic materials within or on the surface of the framework, ${ }^{20-22}$ a variety of functional molecules can be attached to the silica surface via silane linkers. ${ }^{23}$

Mesoporous silica spheres $(100-200 \mathrm{~nm}$ ) were synthesized around the iron oxide nanocrystals by following a modification of the procedures described by Kim et al. and Fan et al. ${ }^{17-19}$ In this procedure, the silica source tetraethylorthosilicate (TEOS) was added into the aqueous solution containing CTAB-coated nanocrystals, $C T A B$, and sodium hydroxide. The interaction between the hydrolyzed TEOS molecules, the CTABcoated nanocrystals, and the free surfactant micelles helped promote the base-catalyzed condensation of TEOS to form the mesostructure. The morphology of the iron oxide-mesoporous silica nanoparticles (NPs) is highly dependent upon the temperature of the solution. When the temperature was too low $\left(<65^{\circ} \mathrm{C}\right)$, slow silica formation resulted in larger-sized materials which consisted of mostly structured mesoporous silica particles with the iron oxide clusters situated on the edges of the silica particles (Figure S-2). As a result, it was necessary to form the NPs at higher temperature, with vigorous stirring and dilute precursor solution, all of which have been used to synthesize mesostructured particles in the nanometer range. ${ }^{24,25}$ However, if the temperature was greater than $80^{\circ} \mathrm{C}$, the mesoporous silica tended to coalesce and form large clumps of materials. At the optimum temperature range $\left(65-80^{\circ} \mathrm{C}\right)$, spherical, 100-200 nm diameter NPs were formed (Figures 3 and S-3). The transmission electron microscope (TEM) images show the dark iron oxide nanocrystals at the center of the NPs and also the 2D hexagonal mesoporous silica structure.

The method involving aqueous transfer of the hydrophobic nanocrystals to aqueous solution and the synthe- 
sis of the nanocrystalsmesoporous silica NPs can be applied to other materials as well. By using this general procedure, we were also able to incorporate gold and silver nanocrystals at the center of the mesoporous silica particles. The hydrophobic dodecanethiol-capped gold nanocrystals ${ }^{26}$ and oleylamine-capped silver nanocrystals ${ }^{27}$ (Figures S-4 and S-6) were first coated with CTAB by using similar procedures. The resulting gold and
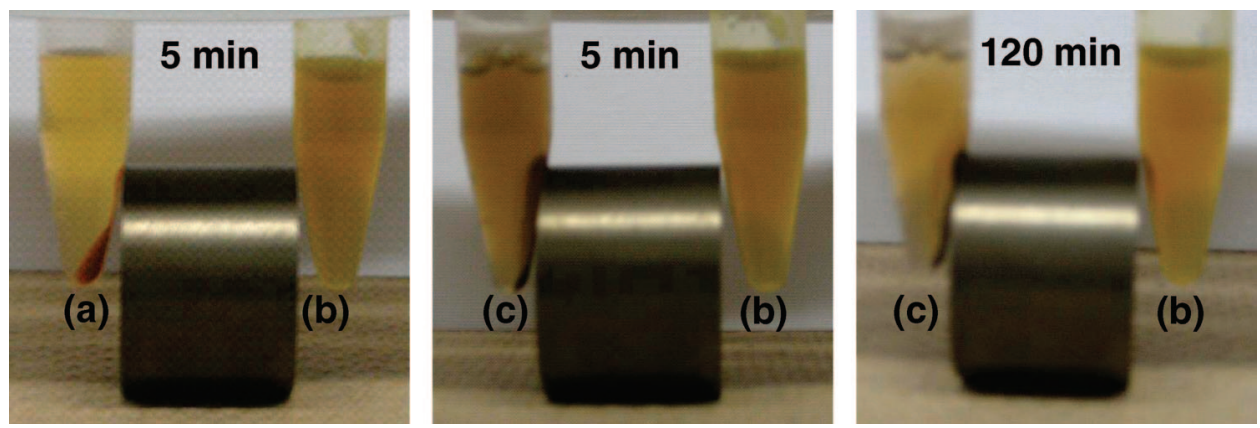

Figure 4. Aqueous suspension of NPs modified with phosphonate (b) compared with those of calcined NPs (a) and NPs without phosphonate (c). After the NPs were dried, they were redispersed in water (5 mg/ $\mathrm{mL}$ ), sonicated thoroughly, and placed next to the magnet. Unlike the other two NPs (a and c), the phosphonate-modified NPs (b) were highly dispersed in the solution and remained suspended even in the presence of the magnetic field. After a longer period of time, the phosphonate-modified NPs were collected by the magnet (right). silver nanocrystals encapsulated within the mesoporous silica NPs are shown in Figures S-5 and S-6, in which the noble metal nanocrystals appear as dense, dark materials at the center of the NPs in the TEM images.

Fluorescent dye molecules were functionalized onto the iron oxide-mesoporous silica NPs using a cocondensation method. Fluorescein isothiocyanate (FITC) was first conjugated with aminopropyltriethoxysilane, and the product, along with TEOS, was then added into the solution containing the starting precursors in order to incorporate fluorescein along the pore walls and particle surface. ${ }^{25,28,29}$ This modification to the particles introduced the fluorescence functionality without affecting the size and shape of the materials, and it enabled the cellular uptake of the NPs to be monitored by fluorescence microscopy.

Pore Characterization. In order to load cargo molecules such as drugs into the NPs, the structure-directing CTAB surfactants must be removed from the mesopores. The typical methods of either calcining or heating the materials in acidic alcohol to extract the surfactants were not suitable. Calcination not only destroys the fluorescent dyes along with any organic surface modifications but also causes irreversible particle aggregation, which makes the materials poorly dispersible in water (Figure 4). Solvent extraction with a mixture of alcohol and hydrochloric acid dissolves the iron oxide nanocrystals. ${ }^{30}$ To circumvent these problems, an ionexchange procedure using ammonium nitrate was used to remove the surfactants from the materials, as confirmed by the FTIR spectra (Figure S-7). 22,31

$\mathrm{X}$-ray diffraction (XRD) analysis and nitrogen adsorption-desorption experiments were conducted on the NPs after the CTAB removal to investigate the porosity. The low-angle XRD pattern of the solventextracted NPs shows a $d$-spacing of approximately 4 $\mathrm{nm}$ (Figure S-8). The adsorption-desorption isotherm (Figure S-9) can be classified as a type IV isotherm according to the IUPAC nomenclature and is typically observed for structured mesoporous materials. ${ }^{24,32}$ The Barret-Joyner-Halenda (BJH) method was used to cal- culate the pore size distribution, yielding an average calculated pore diameter of approximately $3 \mathrm{~nm}$

(Figure S-10).

Aggregation of Particles. Previously, we have shown that mesoporous silica can store water-insoluble drugs within the pores without releasing them in aqueous solution due to the hydrophobic nature of the molecules. $^{29}$ In order to load the hydrophobic molecules into the pores, the particles were suspended in a DMSO solution of the molecules. When the iron

oxide-mesoporous silica NPs were taken out of the DMSO suspension, dried, and added to water, they became poorly dispersed and settled to the bottom quickly. This irreversible aggregation is caused by the interparticle hydrogen-bonding interaction between the surface silanol groups and can be prevented by grafting hydrophilic molecules on the surfaces. ${ }^{33-36}$ The surfaces of the NPs were modified shortly after the particle formation with hydrophilic trihydroxysilylpropyl methylphosphonate to prevent the interparticle aggregation. The dispersibility of the NPs with or without the phosphonate modification was similar if the materials were constantly suspended in solution. However, once the materials were dried and redispersed in aqueous solution, the difference between the NPs with and without phosphonate modification was noticeable (Figure 4). Without the surface modification, the NPs aggre-
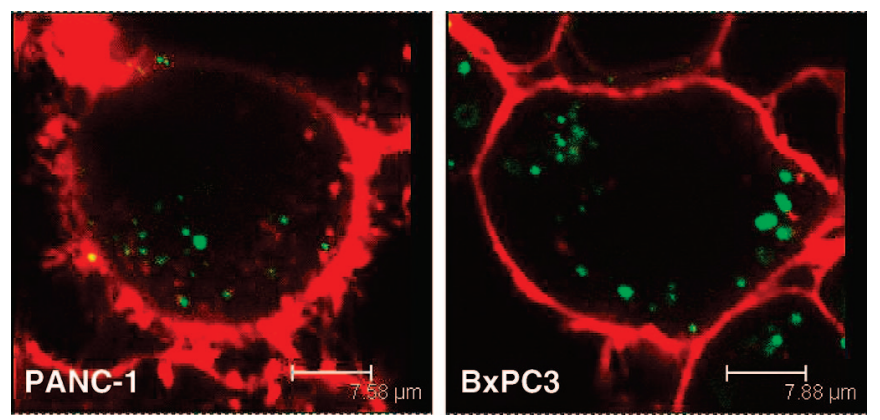

Figure 5. Fluorescence microscopy images of the nanoparticle uptake by human pancreatic cancer cells PANC-1 and BxPC3. The cell membranes (red fluorescence) were stained with WGA, and the clusters of NPs (green fluorescence) were modified with FITC. 


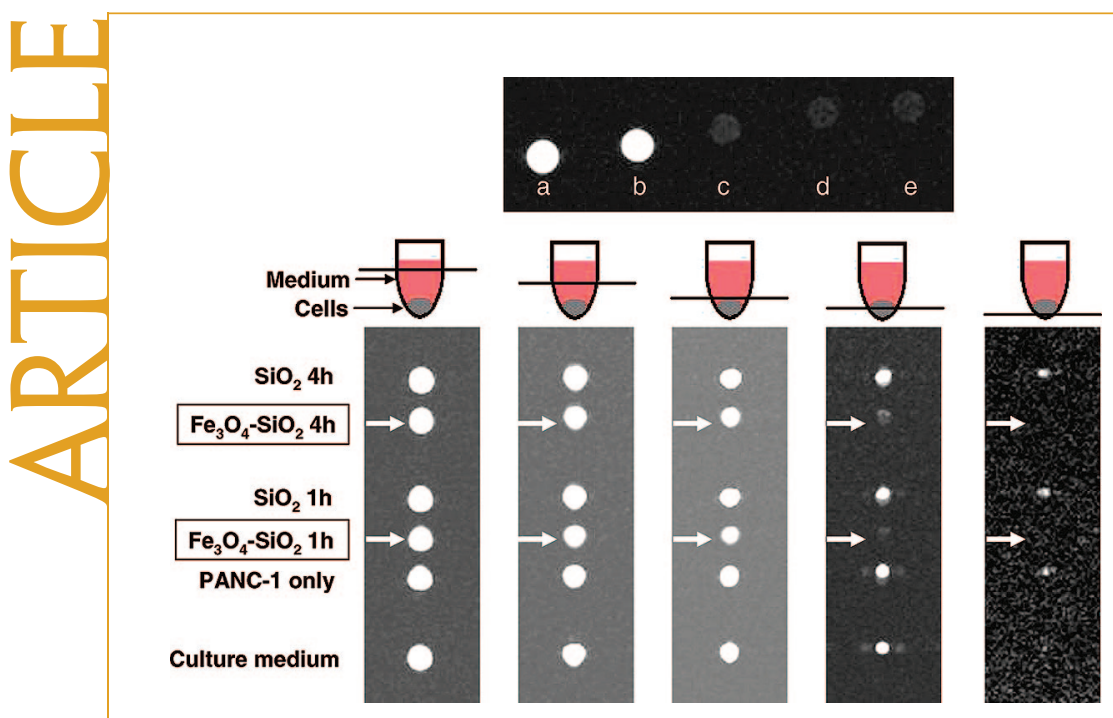

Figure 6. (Top) T2-weighted MR images of (a) water, (b) plain mesoporous silica NPs $(2 \mathrm{mg} / \mathrm{mL})$, and iron oxide-mesoporous silica NPs at (c) 4 , (d) 2, and (e) $1 \mathrm{mg} /$ $\mathrm{mL}$. (Bottom) Cross-section T2-weighted MR images of the centrifuge tubes at different tube heights. PANC-1 cells that were treated with iron oxide-mesoporous silica NPs (labeled with arrows) appeared dark compared to the other samples.

gated and could easily be collected by the magnet, similar to the calcined NPs. The phosphonate-modified $\mathrm{NPs}$, on the other hand, were very stable and remained suspended in the solution. Over a longer period of $2 \mathrm{~h}$, some of the phosphonate-modified NPs were collected by the neodymium magnet, showing that they can be manipulated by an external magnetic field.

Particle Uptake by Cells. The cellular uptake of the NPs was confirmed on two different pancreatic cancer cell lines, PANC-1 and BxPC3. The NPs were able to enter the cells within 30 min without causing any observed toxicity. As shown in Figure 5, the clusters of NPs (green fluorescence) were located within the cells and not on the cell membranes (red fluorescence, WGA-Alexa Fluor 594 stain). Additionally, the treated cells were examined cross-sectionally by confocal fluorescence microscopy in order to confirm that the NPs were indeed internalized by the cells and not simply bound on the surface membrane.

MR Imaging. In order to determine whether these NPs could be used as contrast agents in MR imaging, their contrast effect in solution and inside the cells was tested using a clinical MRI instrument. Different concentrations

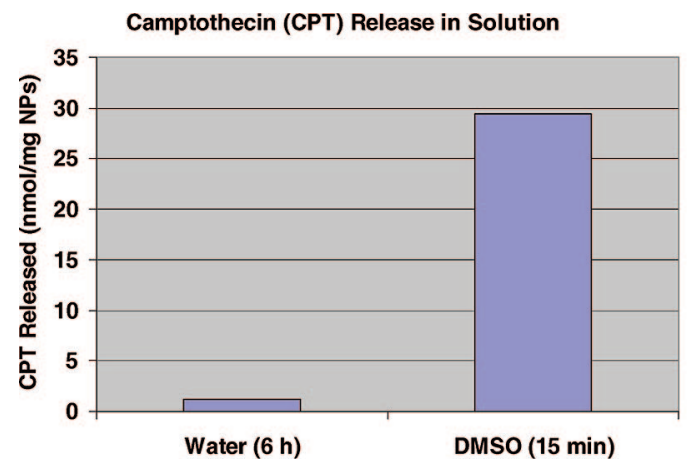

of the aqueous NPs suspension $(1-4 \mathrm{mg} / \mathrm{mL}$ ) were placed in the centrifuge tubes. For further comparison, plain mesoporous silica NPs (without the iron oxides) were also tested to confirm that it was not the silicate materials that caused the contrast. Superparamagnetic iron oxide nanocrystals are used as contrast agents in MRI because of their negative enhancement effect on T2-weighted sequences. ${ }^{37}$ As a result, the tubes containing the iron oxide-mesoporous silica NPs appeared dark in the T2-weighted MR image (Figure 6). On the other hand, the tubes that contained water and plain mesoporous silica NPs remained bright and indistinguishable.

To observe the contrast effect inside the cells, PANC-1 cells were first treated with the NPs for 1 or $4 \mathrm{~h}$ before being washed and collected in Dulbecco's modified Eagle's medium (DMEM) in 0.2 $\mathrm{mL}$ centrifuge tubes. The control samples consisted of the DMEM, the untreated cells, and the cells treated with plain mesoporous silica NPs. Several T2-weighted images of the cross sections were taken, going from the top of the tubes (containing the media) to the bottom of the tubes (containing the cells) (Figure 6). The tubes containing the control samples were comparable in brightness, whereas the tubes containing the cells treated with the iron oxide-mesoporous silica NPs appeared dark because of the decrease in T2 relaxation. These results show that the NPs can be used as MR contrast agents in solution and inside cells.

Drug Delivery. The NPs were used to store and deliver water-insoluble anticancer drugs into cells. The materials were loaded with either camptothecin (CPT) or paclitaxel (TXL) by soaking them in a concentrated drug-DMSO solution. The drug-loaded NPs were collected by centrifugation to remove the supernatant and dried under vacuum before being resuspended in aqueous solution. By using UV/vis absorption spectroscopy, it was observed that only $4 \%$ of the stored drug molecules were released into the supernatant when the drug-loaded NPs were dispersed in aqueous solution and left in suspension for $6 \mathrm{~h}$. However, once the drugloaded NPs were again dispersed in DMSO or metha-

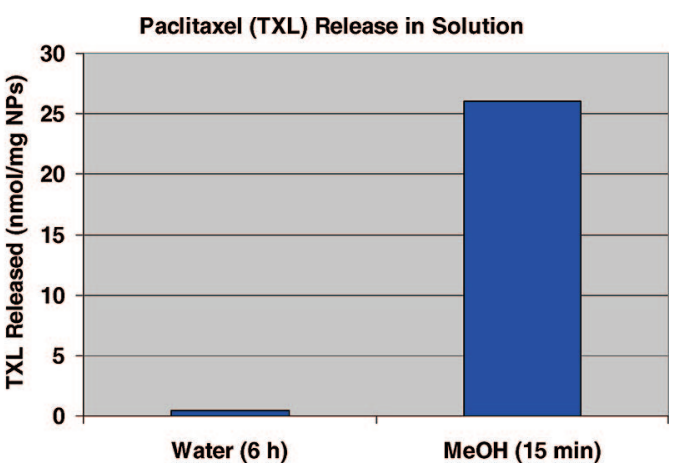

Figure 7. UV/vis absorption measurements show that most of the water-insoluble drug molecules were trapped inside the pores when the NPs were dispersed in water, but they were quickly released in organic solvents. 
nol, all of the drugs came out of the mesopores and were observed in the supernatant (Figure 7). Based on absorption measurements, approximately $30 \mathrm{nmol}$ of drug molecules were stored inside $1 \mathrm{mg}$ of the NPs.

The efficacy of these drug-loaded NPs was tested on the pancreatic cancer cell lines PANC- 1 and BxPC3. The NPs alone were not toxic to the cells at the concentrations used in the experiment, but the drug-loaded NPs caused observable cytotoxicity to both cell lines (Figure 8). We postulated that the water-insoluble drugs were released from the mesopores when the NPs had entered the cells. Furthermore, the aqueous suspensions of the drug-loaded NPs were stable for long periods of time since they retained their cytotoxicity after over 2 months of storage at $4{ }^{\circ} \mathrm{C}$. Based on these results, the NPs can potentially be used as a vehicle to store and deliver anticancer drugs that are both highly toxic and water-insoluble into different types of cancer cells.

Targeting of Cancer Cells. Folic acid was used as the targeting component for this study because $\alpha$-folate receptor is observed to be up-regulated in various types of human cancers. ${ }^{38,39}$ Since the coating with phosphonate groups was done during the particle synthesis, there still remained many surface silanol groups at the pore orifices after the CTAB had been removed, which can be used for further surface modification. ${ }^{40-42}$ The amide linkage between the carboxyl group on the folic acid and the amine group on the aminopropyltriethoxysilane was first formed before grafting the

folate-silanes onto the surface of the NPs. ${ }^{28,43}$ The same batch of materials was used for the in vitro comparison between the NPs and the folate-modified NPs to avoid problems of batch-to-batch variability.

The effect of folic acid modification on the cellular uptake of NPs was studied with the cancer cells PANC-1 and human foreskin fibroblasts (HFF). Western blot and reverse transcription polymerase chain reaction (RT-PCR) confirmed the overexpression of $\alpha$-folate receptor on PANC-1 cells at both protein and mRNA levels, but not on the HFF (Figure 9). Although the cellular uptake of NPs was observed with both cell lines, folate modification on the NPs increased the particle uptake by the PANC-1 more than 2-fold, but not by the HFF (Figure 10). These results corroborated the overexpression of folate receptor on PANC-1 cells, which may facilitate the recognition of the folate-modified NPs and increase the uptake through folate receptor-mediated endocytosis. $^{43,44}$

Folic acid modification to the CPT-loaded NPs can selectively increase the delivery of drugs to the cells that overexpress $\alpha$-folate receptor. Because the NPs can enter both PANC-1 and HFF, the cytotoxicity of the CPTloaded NPs was observed for both cell lines. However, there was a considerable increase in the cytotoxicity of folate-modifed CPT-loaded NPs to PANC-1 cells (Figure 11), which correlated with the aforementioned enhanced particle uptake. More importantly, the cytotox-

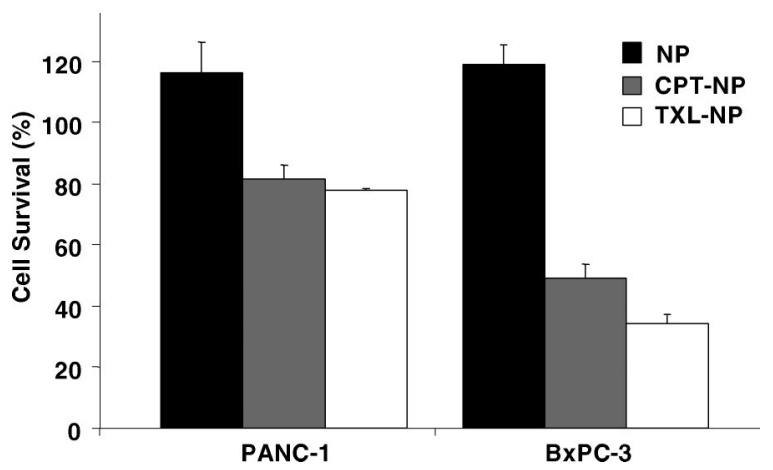

Figure 8. Cell growth inhibition assay for the drug-loaded NPs. Human pancreatic cancer cells PANC- 1 and BxPC-3 were treated for $24 \mathrm{~h}$ with nanoparticles (NP), camptothecin-loaded nanoparticles (CPT-NP), or paclitaxel-loaded nanoparticles (TXL-NP). The concentration of the NPs used was $20 \mu \mathrm{g} / \mathrm{mL}$.

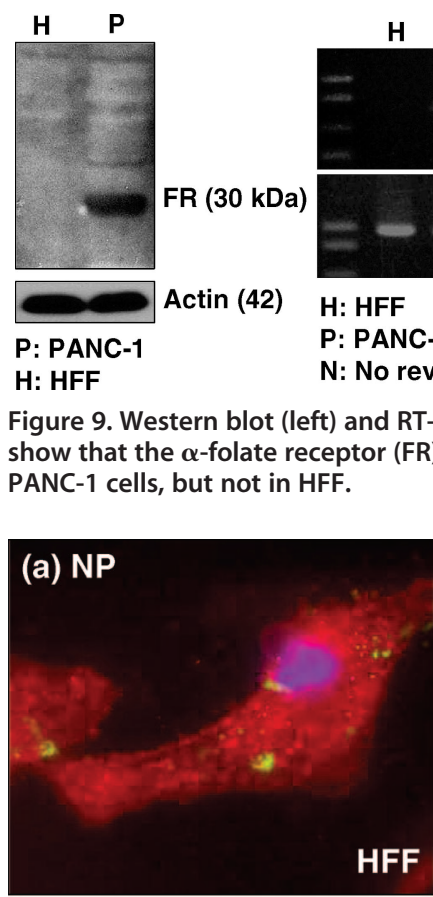

H $\quad \mathrm{P} \quad \mathrm{N}$

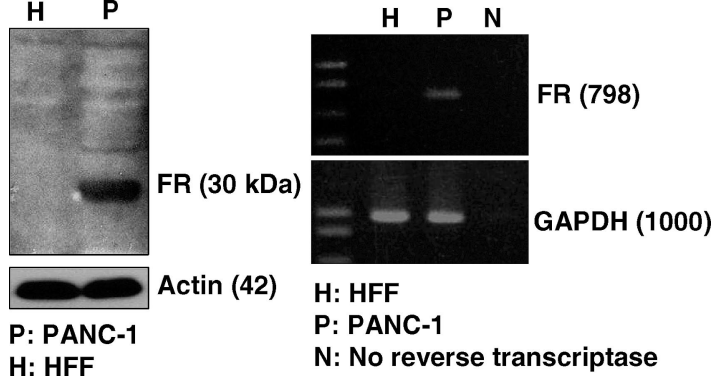

Figure 9. Western blot (left) and RT-PCR (right) analyses show that the $\alpha$-folate receptor (FR) was overexpressed in
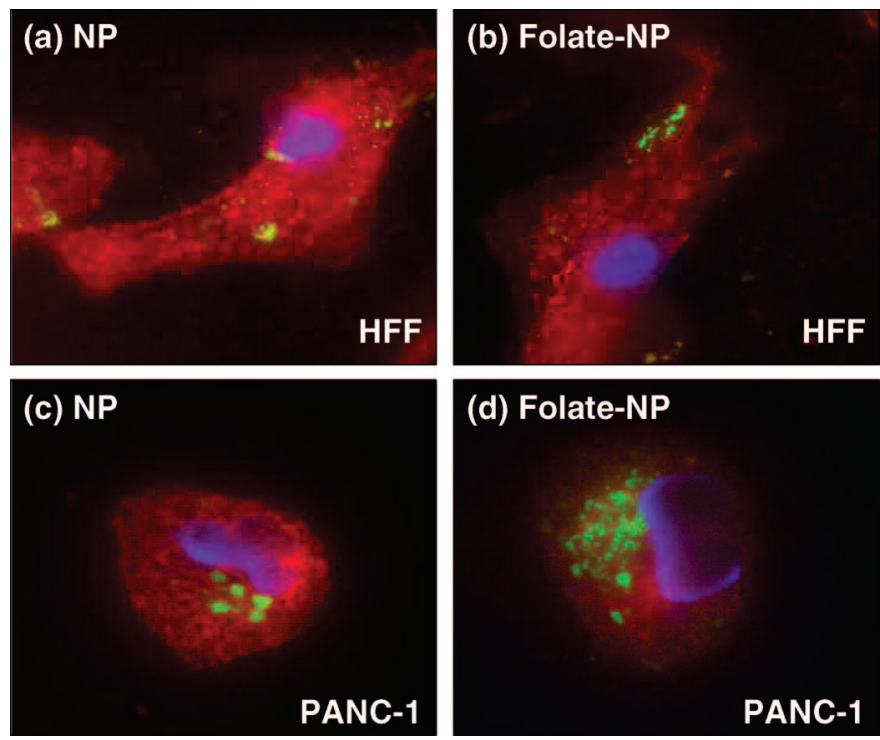

Figure 10. Fluorescence microscopy images showing the effect of folic acid modification on the NPs (green fluorescence). The cell nuclei were stained with DAPI (blue fluorescence), and the membranes were stained with WGA (red fluorescence). Top figures: HFF treated with (a) NPs and (b) folate-modified NPs. Bottom figures: PANC-1 treated with (c) NPs and (d) folate-modified NPs. Increased uptake of the folate-modified NPs was observed with the PANC-1 cells (overexpressed folate receptor) but not with the HFF.

icity between the folate-modified and the unmodified CPT-loaded NPs was similar for the HFF since these cells do not overexpress the receptors. The result showed that folic acid modification to the NPs can increase the 


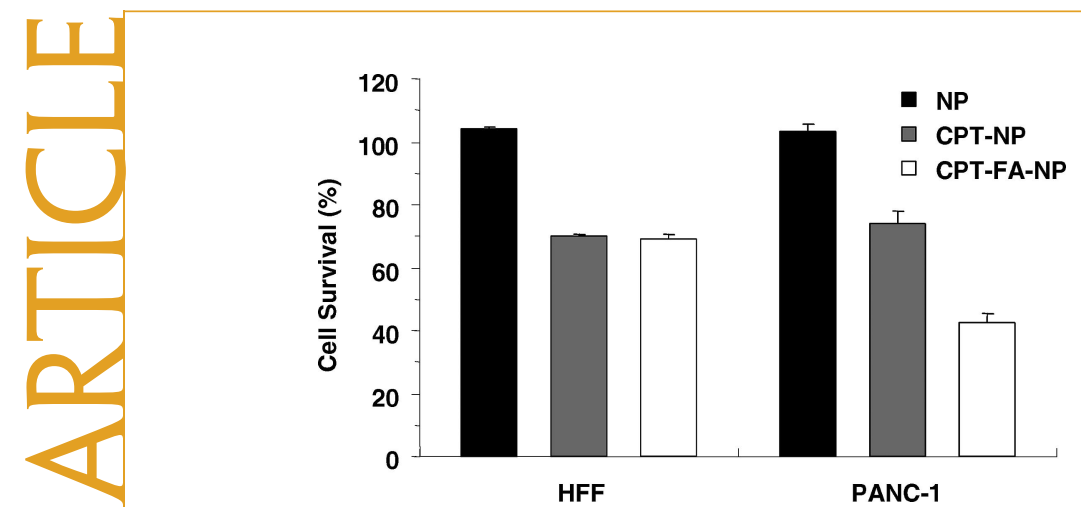

Figure 11. Cell growth inhibition assay of the folate-modified materials. The cells were treated for $\mathbf{2 4} \mathrm{h}$ with nanoparticles only (NP), camptothecin-loaded nanoparticles (CPT-NP), or camptothecin-loaded nanoparticles modified with folic acid (CPT-FA-NP). The enhanced uptake of NPs by PANC-1 cells through folate modification led to an increase in the delivery of camptothecin. This effect was not observed on HFF, which do not overexpress folate receptors. The concentration of the NPs used was $20 \mu \mathrm{g} / \mathrm{mL}$.

particle uptake and deliver more drugs to the cancer cells but not to the non-cancerous fibroblasts.

\section{SUMMARY}

We have synthesized multifunctional iron oxide-mesoporous silica nanoparticles that are detectable by both MR imaging and optical methods. Fluorescence imaging and cell viability assays show that the nanoparticles deliver water-insoluble drug molecules into cells and have increased specificity toward cancer cells. The chemical reagents used for the particle synthesis are inexpensive and nontoxic (e.g., $\mathrm{FeCl}_{3}$, TEOS, folic acid), and the simple procedures are suitable for large-scale production. The imaging functionalities allow non-invasive tracking of the nanoparticles within the body. Targeted drug delivery using the folate-modified nanoparticles will be useful for treating cancer while minimizing the toxicity to the surrounding normal tissues. Combining the facile procedures and the targeting aspect with the dual-imaging capability, these nanoparticles may prove valuable for simultaneous imaging and drug delivery purposes.

\section{METHODS}

Synthesis of Iron 0xide Nanocrystals. The iron oxide nanocrystals (NCs) were synthesized by the thermal decomposition of iron-oleate complexes in a solution of oleic acid surfactants and octadecene solvent. ${ }^{13}$ First, $2.2 \mathrm{~g}$ of iron(III) chloride hexahydrate (Sigma, $98 \%$ ) and $7.4 \mathrm{~g}$ of sodium oleate $(\mathrm{TCl}, 95 \%)$ were dissolved in a mixture of $16.3 \mathrm{~mL}$ of absolute ethanol and 12.2 $\mathrm{mL}$ of water and mixed with $28.5 \mathrm{~mL}$ of hexane. The solution was refluxed for $4 \mathrm{~h}$. The mixture was then washed with water several times in a separatory funnel, and the hexane was removed from the mixture by using rotary evaporation. The synthesized iron-oleate complex was then dried under vacuum overnight. One gram of the iron-oleate complex was dissolved in a solution of $177.3 \mu \mathrm{L}$ of oleic acid (Aldrich, $90 \%$ ) and $7.1 \mathrm{~mL}$ of octadecene (Aldrich, 90\%). The mixture was placed under vacuum and heated at $80^{\circ} \mathrm{C}$ for $30 \mathrm{~min}$. It was then stirred vigorously under nitrogen flow, heated to $320^{\circ} \mathrm{C}$ at a rate of $3^{\circ} \mathrm{C} / \mathrm{min}$, and kept at that temperature for $1 \mathrm{~h}$. After the mixture had cooled to room temperature, $5 \mathrm{~mL}$ of hexane was added, and the NCs were precipitated by adding an excess of ethanol. The NCs were separated from the solution by centrifugation. The NCs were then washed twice in a solution of 1:3 hexane-ethanol and dried under vacuum.

Mesoporous Silica Formation. The dried oleate-capped iron oxide $\mathrm{NCs}$ were dissolved in chloroform. Two milliliters $(10-20 \mathrm{mg} /$ $\mathrm{mL}$ ) of the NCs solution was mixed with $0.4 \mathrm{~g}$ of cetyltrimethylammonium bromide (CTAB, Aldrich, $95 \%$ ) and $20 \mathrm{~mL}$ of water. The mixture was then sonicated and stirred vigorously, and the chloroform solvent was boiled off from the solution. The aqueous CTAB-iron oxide NCs solution was filtered through a 0.44 $\mu \mathrm{m}$ syringe filter to remove any large aggregates or contaminants. One milligram of fluorescein isothiocyanate (FITC, Sigma, 90\%) was dissolved in $545 \mu \mathrm{L}$ of absolute ethanol and mixed with $2.2 \mu \mathrm{L}$ of aminopropyltriethoxysilane (APTS, Aldrich, 99\%) for $2 \mathrm{~h}$. Five milliliters of the aqueous CTAB-stabilized NCs solution was added into a solution of $43 \mathrm{~mL}$ of distilled water and 350 $\mu \mathrm{L}$ of sodium hydroxide $(2 \mathrm{M})$ and heated to $80^{\circ} \mathrm{C}$. For higher concentration of iron oxide materials, the solution may need to be heated at lower temperature $\left(65-70^{\circ} \mathrm{C}\right)$ in order to avoid the coalescence of the mesoporous silica in forming large clumps of materials. After the temperature had stabilized, $0.6 \mathrm{~mL}$ of the ethanolic FITC - APTS solution was mixed with $0.5 \mathrm{~mL}$ of tetraethylorthosilicate and added slowly into the aqueous solution containing the CTAB-stabilized NCs. After 15 min of stirring, $127 \mu \mathrm{L}$ of 3-(trihydroxysilyl)propyl methylphosphonate (Aldrich, 42\%) was added into the mixture, and the solution was stirred for another $2 \mathrm{~h}$. The synthesized materials were centrifuged and washed with methanol. The CTAB surfactants were removed from the mesopores by dispersing the as-synthesized materials in a solution of $160 \mathrm{mg}$ of ammonium nitrate (Fisher) and $60 \mathrm{~mL}$ of $95 \%$ ethanol and heating the mixture at $60^{\circ} \mathrm{C}$ for $15 \mathrm{~min}$. The materials were then centrifuged and washed with ethanol.

Gold - and Silver-Mesoporous Silica. Gold NCs were synthesized by following the Brust method. ${ }^{26}$ First, $180 \mathrm{mg}$ of gold(III) chloride trihydrate (Aldrich, $99.9 \%$ ) was dissolved in $15.3 \mathrm{~mL}$ of water and mixed with $40.6 \mathrm{~mL}$ of toluene solution containing $1.1 \mathrm{~g}$ of tetraoctylammonium bromide (Aldrich, 98\%). The solution was stirred vigorously for 30 min before addition of $102.3 \mu \mathrm{L}$ of dodecanethiol (Aldrich, 98\%). Next, $12.7 \mathrm{~mL}$ of aqueous solution of $192.1 \mathrm{mg}$ of sodium borohydride (Alfa Aesar, 97\%) was added slowly to the mixture. After further stirring for $3 \mathrm{~h}$, the aqueous layer was removed using a separatory funnel, and the toluene was removed using rotary evaporation. The solids were dissolved in a minimal amount of toluene, precipitated with absolute ethanol, and collected by centrifugation. After the process was repeated two more times, the solids were dried under vacuum.

Silver NCs were synthesized by following the method developed by Hiramatsu and Osterloh. ${ }^{27}$ First, $50 \mathrm{mg}$ of silver acetate (Aldrich, 99\%) was dissolved in $2.5 \mathrm{~mL}$ of oleylamine (Aldrich, $70 \%$ ) and added quickly into a boiling toluene solution. The mixture was refluxed and stirred vigorously for $12 \mathrm{~h}$. Most of the solvent was removed using rotary evaporation until $\sim 5 \mathrm{~mL}$ remained in the container. The silver NCs were precipitated by adding methanol into the mixture and recovered by centrifugation. The process was repeated two more times using a minimal amount of hexane and an excess of methanol before drying the solids under vacuum.

To synthesize the gold-mesoporous silica NPs and silver-mesoporous silica NPs, a procedure similar to that used to make the iron oxide-mesoporous silica NPs was followed.

Folic Acid Modification. To attach folic acid to the iron oxide-mesoporous silica NPs, $20 \mathrm{mg}$ of the materials (after removing the $C T A B$ using the ion-exchange method) were washed with dimethyl sulfoxide (DMSO) and resuspended in DMSO. In a flask, $0.1 \mathrm{mg}$ of folic acid (Sigma, 98\%) and $0.05 \mu \mathrm{L}$ of APTS were mixed in $1 \mathrm{~mL}$ of DMSO. Next, $0.03 \mathrm{mg}$ of $\mathrm{N}$-hydroxysuccinimide (Aldrich, $98 \%$ ) and $0.05 \mathrm{mg}$ of 1-(3-dimethylaminopropyl)-3ethylcarbodiimide hydrochloride (Alfa Aesar, 98\%) were added 
into the mixture and stirred for $2 \mathrm{~h}$. In a separate flask containing $4 \mathrm{~mL}$ of toluene and the NPs-DMSO suspension, the folate-APTS solution was added, and the mixture was stirred for $20 \mathrm{~h}$ at room temperature. The materials were recovered by centrifugation, washed twice with toluene, and dried under vacuum.

Drug Loading. The modified materials were loaded with either camptothecin (CPT, Sigma, 95\%) or paclitaxel (TXL, Sigma) by incubating $10 \mathrm{mg}$ of the materials in a solution of $1 \mathrm{mg}$ of drugs and $0.25 \mathrm{~mL}$ of DMSO for $4 \mathrm{~h}$. After the drug-loaded NPs were removed from the suspension by centrifugation and the supernatant was removed completely, the materials were then dried under vacuum. The drug-loaded NPs were washed and sonicated with water before being resuspended in aqueous solution

In order to determine the amount of drugs that were inside the NPs, the aqueous drug-loaded NPs suspension was incubated at 4 ${ }^{\circ} \mathrm{C}$ for $6 \mathrm{~h}$ before centrifugation to show that the drugs were not being slowly released from the mesopores. The resulting supernatant was mixed with the previous supernatant solution from the washing process and measured using UV/vis absorption spectroscopy. The drug-loaded NPs pellet was resuspended and sonicated in DMSO (or methanol for TXL-loaded NPs) and collected by centrifugation. The process was repeated two more times ( $\sim 15 \mathrm{~min}$ total time) to ensure that the drugs were completely removed from the pores. The DMSO (or methanol) supernatants were then measured using UV/vis absorption.

Cell Culture. Human cancer cell lines PANC-1 and BxPC3 were obtained from the American Type Culture Collection, and human foreskin fibroblasts (HFF) were a generous gift from Dr. Peter Bradley at UCLA. The cells were maintained in Dulbecco's modified Eagle's medium (DMEM, GIBCO) supplemented with $10 \%$ fetal calf serum, $2 \%$ L-glutamine, $1 \%$ penicillin, and $1 \%$ streptomycin stock solutions. The media were changed every three days, and the cells were passaged by trypsinization before confluence.

Fluorescence Microscopy. The cellular uptake of the NPs was confirmed by fluorescence microscopy. The cells were incubated in an eight-well cell culture chamber with the NPs and then washed with DMEM and PBS to remove the NPs that did not enter the cells. The cells were then stained with DAPI solution and/or WGA-Alexa Fluor 594 before being monitored using the fluorescence microscope.

Cell Viability Assay. The cytotoxicity assay was performed by using a cell-counting kit from Dojindo Molecular Technologies, Inc. Cells were seeded in 96-well plates (5000 cells/well) and incubated in fresh culture medium at $37{ }^{\circ} \mathrm{C}$ in a $5 \% \mathrm{CO}_{2} / 95 \%$ air atmosphere for $24 \mathrm{~h}$. The cells were then washed with PBS, and the medium was replaced with a fresh medium containing the NPs or the drug-loaded NPs. After $24 \mathrm{~h}$, the cells were washed with PBS and incubated in fresh medium for an additional $48 \mathrm{~h}$. The cells were washed with PBS and incubated in DMEM with $10 \%$ WST- 8 solution for another $2 \mathrm{~h}$. The absorbance of each well was measured at $450 \mathrm{~nm}$ with a plate reader. Since the absorbance is proportional to the number of viable cells in the medium, the viable cell number was determined by using a previously prepared calibration curve (Dojindo Co.).

Western Blot Analysis. Cell lysate was separated by gel electrophoresis on a polyacrylamide gel containing sodium dodecyl sulfate and then transferred to nitrocellulose membranes. The membranes were blocked with Tris-buffered saline (TBS) containing 5\% $(\mathrm{w} / \mathrm{v})$ skimmed milk. After being washed with TBS containing $0.1 \%$ Tween 20 (Sigma), the membranes were incubated overnight at room temperature with $\alpha$-folate receptor (F-15) antibody (Santa Cruz Biotechnology) diluted with TBS. After being washed, the membranes were incubated for $2 \mathrm{~h}$ at room temperature with the second antibody (Santa Cruz Biotechnology). Bands were detected with an ECL system (Amersham Pharmacia Biotech.)

Reverse Transcription Polymerase Chain Reaction (RT-PCR). RT-PCR was performed using a platinum taq DNA polymerase highfidelity RT-PCR kit. The cells were harvested from culture dishes. RNA was extracted using TRIzol reagents (Invitrogen), and $1 \mu \mathrm{g}$ of RNA was reverse-transcribed. The resulting cDNAs were amplified by PCR reaction using primers for human folate receptor (forward, AACACAGCTGCTGCTCCTTCTAGT; reverse, AACAGGGCAGGGATITCCAGGTAT). The PCR reaction was conducted for 40 cycles. Each cycle consisted of $30 \mathrm{~s}$ at $94{ }^{\circ} \mathrm{C}, 30 \mathrm{~s}$ at $57{ }^{\circ} \mathrm{C}$, and $1 \mathrm{~min}$ at $72{ }^{\circ} \mathrm{C}$. The reaction products were electrophoresed in $1 \%$ TAB agarose gel. The gel was stained by ethidium bromide and then photographed.

Magnetic Resonance (MR) Imaging. The MR imaging experiments were performed on a Siemens Avanto 1.5-T MR system. An extremity coil was used for the data acquisition, and the pulse sequence used was a T2-weighted turbo spin-echo sequence with the following parameters: TR $=4620 \mathrm{~ms}$, slice thickness $=3 \mathrm{~mm}, \mathrm{TE}=98$ $\mathrm{ms}$, field of view $=157 \times 180 \mathrm{~mm}$, number of acquisitions $=1$. For the experiments to observe the MR contrast effect of the NPs within the cells, PANC-1 cells were incubated with either the iron oxide-mesoporous silica NPs or plain mesoporous silica NPs ${ }^{29}$ for 1 and $4 \mathrm{~h}$ periods, trypsinized, and then placed in a $0.2 \mathrm{~mL} \mathrm{PCR}$ tube. Each tube contained approximately $10^{5}$ cells.

Acknowledgment. We thank Jae-Hyun Lee and Prof. Jinwoo Cheon for helpful discussions, Eunwoo Choi and Prof. Omar M Yaghi for nitrogen adsorption measurements, and Michael Lo and Prof. Miguel Garcia-Garibay for assistance with FTIR measurements. This work was supported by the National Science Foundation (DMR 0346601), the U.S. National Institutes of Health (CA32737), U.S. Public Health Service Grants (ES10553, RO1 ES10253, and RO1 ES015498), the U.S. EPA STAR award (RD83241301) to the Southern California Particle Center, and the University of California (UC) Lead Campus for Nanotoxicology Training and Research, funded by the UC TSR\&TP. Fluorescence microscopy was performed at the CNSI Advanced Light Microscopy/Spectroscopy Shared Facility at UCLA. This work has not been subjected to the EPA for peer and policy review.

Supporting Information Available: TEM of iron oxide nanocrystals, the gold - and silver-mesoporous silica NPs, and XRD and nitrogen adsorption - desorption analysis. This material is available free of charge via the Internet at http://pubs.acs.org.

\section{REFERENCES AND NOTES}

1. Georganopoulou, D. G.; Chang, L.; Nam, J.-M.; Thaxton, C. S.; Mufson, E. J.; Klein, W. L.; Mirkin, C. A. Nanoparticle-Based Detection in Cerebral Spinal Fluid of a Soluble Pathogenic Biomarker for Alzheimer's Disease. Proc. Natl. Acad. Sci. U.S.A. 2005, 102, 2273-2276.

2. Gao, X.; Cui, Y.; Levenson, R. M.; Chung, L. W. K.; Nie, S. In Vivo Cancer Targeting and Imaging with Semiconductor Quantum Dots. Nat. Biotechnol. 2004, 22, 969-976.

3. Wu, X.; Liu, H.; Liu, J.; Haley, K. N.; Treadway, J. A.; Larson, J. P.; Ge, N.; Peale, F.; Bruchez, M. P. Immunofluorescent Labeling of Cancer Marker Her2 and Other Cellular Targets with Semiconductor Quantum Dots. Nat. Biotechnol. 2002, 21 , 41-46.

4. Lee, J.-H.; Huh, Y.-M.; Jun, Y.-W.; Seo, J.-W.; Jang, J.-T.; Song, H.-T.; Kim, S.; Cho, E.-J.; Yoon, H.-G.; Suh, J.-S.; et al. Artificially Engineered Magnetic Nanoparticles for Ultra-Sensitive Molecular Imaging. Nat. Med. 2007, 13, 95-99.

5. Na, H. B.; Lee, J. H.; An, K.; Park, Y. I.; Park, M.; Lee, I. S.; Nam, D.-H.; Kim, S. T.; Kim, S.-H.; Kim, S.-W.; et al. Development of a T1 Contrast Agent for Magnetic Resonance Imaging Using MnO Nanoparticles. Angew. Chem., Int. Ed. 2007, 46, 53975401.

6. Slowing, I. I.; Trewyn, B. G.; Lin, V. S.-Y. Mesoporous Silica Nanoparticles for Intracellular Delivery of MembraneImpermeable Proteins. J. Am. Chem. Soc. 2007, 129, 88458849.

7. Huang, X.; El-Sayed, I. H.; Qian, W.; El-Sayed, M. A. Cancer Cell Imaging and Photothermal Therapy in the Near-Infrared Region by Using Gold Nanorods. J. Am. Chem. Soc. 2006, 128, 2115-2120.

8. Chen, J.; Wang, D.; Xi, J.; Au, L.; Siekkinen, A.; Warsen, A.; Li, Z. Y.; Zhang, H.; Xia, Y.; Li, X. Immuno Gold Nanocages with Tailored Optical Properties for Targeted Photothermal Destruction of Cancer Cells. Nano Lett. 2007, 7, 1318-1322.

9. Gobin, A. M.; Lee, M. H.; Halas, N. J.; James, W. D.; Drezek, R. A.; West, J. L. Near-Infrared Resonant Nanoshells for Combined Optical Imaging and Photothermal Cancer Therapy. Nano Lett. 2007, 7, 1929-1934. 
10. Kohler, N.; Sun, C.; Fichtenholtz, A.; Gunn, J.; Fang, C.; Zhang, M. Methotrexate-Immobilized Poly(ethylene glycol) Magnetic Nanoparticles for MR Imaging and Drug Delivery. Small 2006, 2, 785-792.

11. Medarova, Z.; Pham, W.; Farrar, C.; Petkova, V.; Moore, A. In Vivo Imaging of siRNA Delivery and Silencing in Tumors. Nat. Med. 2007, 13, 372-377.

12. Jana, N. R.; Chen, Y.; Peng, X. Size- and Shape-Controlled Magnetic ( $\mathrm{Cr}, \mathrm{Mn}, \mathrm{Fe}, \mathrm{Co}, \mathrm{Ni}$ ) Oxide Nanocrystals via a Simple and General Approach. Chem. Mater. 2004, 16, 3931-3935.

13. Park, J.; An, K.; Hwang, Y.; Park, J.-G.; Noh, H.-J; Kim, J.-Y.; Park, J.-H.; Hwang, N.-M.; Hyeon, T. Ultra-Large-Scale Syntheses of Monodisperse Nanocrystals. Nat. Mater. 2004, 3, 891-895.

14. Sun, S.; Zeng, H.; Robinson, D. B.; Raoux, S.; Rice, P. M.; Wang, S. X.; Li, G. Monodisperse $\mathrm{MFe}_{2} \mathrm{O}_{4}(\mathrm{M}=\mathrm{Fe}, \mathrm{Co}, \mathrm{Mn})$ Nanoparticles. J. Am. Chem. Soc. 2004, 126, 273-279.

15. Yu, W. W.; Falkner, J. C.; Yavuz, C. T.; Colvin, V. L. Synthesis of Monodisperse Iron Oxide Nanocrystals by Thermal Decomposition of Iron Carboxylate Salts. Chem. Commun. 2004, 2306-2307.

16. Jun, Y. W.; Huh, Y. M.; Choi, J. S.; Lee, J. H.; Song, H. T.; Kim, S. J.; Yoon, S.; Kim, K. S.; Shin, J. S.; Suh, J. S.; et al. Nanoscale Size Effect of Magnetic Nanocrystals and Their Utilization for Cancer Diagnosis via Magnetic Resonance Imaging. J. Am. Chem. Soc. 2005, 127, 5732-5733.

17. Fan, H.; Yang, K.; Boye, D. M.; Sigmon, T.; Malloy, K. J.; Xu, H.; Lopez, G. P.; Brinker, C. J. Self-Assembly of Ordered, Robust, Three-Dimensional Gold Nanocrystal/Silica Arrays. Science 2004, 304, 567-571.

18. Fan, H.; Gabaldon, J.; Brinker, C. J.; Jiang, Y.-B. Ordered Nanocrystal/Silica Particles Self-Assembled from Nanocrystal Micelles and Silicate. Chem. Commun. 2006, 2323-2325.

19. Kim, J.; Lee, J. E.; Lee, J.; Yu, J. H.; Kim, B. C.; An, K.; Hwang, Y.; Shin, C.-H.; Park, J.-G.; Hyeon, T. Magnetic Fluorescent Delivery Vehicle Using Uniform Mesoporous Silica Spheres Embedded with Monodisperse Magnetic and Semiconductor Nanocrystals. J. Am. Chem. Soc. 2006, 128, 688-689.

20. Jiang, X.; Brinker, C. J. Aerosol-Assisted Self-Assembly of Single-Crystal Core/Nanoporous Shell Particles as Model Controlled Release Capsules. J. Am. Chem. Soc. 2006, 128, 4512-4513.

21. Lee, J.-H.; Jun, Y.-w.; Yeon, S.-I.; Shin, J.-S.; Cheon, J. DualMode Nanoparticle Probes for High-Performance Magnetic Resonance and Fluorescence Imaging of Neuroblastoma. Angew. Chem., Int. Ed. 2006, 118, 8340-8342.

22. Lin, Y. S.; Wu, S. H.; Hung, Y.; Chou, Y. H.; Chang, C.; Lin, M. L.; Tsai, C. P.; Mou, C. Y. Multifunctional Composite Nanoparticles: Magnetic, Luminescent, and Mesoporous. Chem. Mater. 2006, 18, 5170-5172.

23. Wang, L.; Zhao, W.; O'Donoghue, M. B.; Tan, W. Fluorescent Nanoparticles for Multiplexed Bacteria Monitoring. Bioconjugate Chem. 2007, 18, 297-301.

24. Cai, Q.; Luo, Z.-S.; Pang, W.-Q.; Fan, Y.-W.; Chen, X.-H.; Cui, F.Z. Dilute Solution Routes to Various Controllable Morphologies of MCM-41 Silica with a Basic Medium. Chem. Mater. 2001, 13, 258-263.

25. Lin, Y. S.; Tsai, C. P.; Huang, H. Y.; Kuo, C. T.; Hung, Y.; Huang, D. M.; Chen, Y. C.; Mou, C. Y. Well-Ordered Mesoporous Silica Nanoparticles as Cell Markers. Chem. Mater. 2005, 17 4570-4573.

26. Brust, M.; Walker, M.; Bethell, D.; Schiffrin, D. J.; Whyman, R. Synthesis of Thiol-Derivatised Gold Nanoparticles in a TwoPhase Liquid—Liquid System. Chem. Commun. 1994, 801-802.

27. Hiramatsu, H.; Osterloh, F. E. A Simple Large-Scale Synthesis of Nearly Monodisperse Gold and Silver Nanoparticles with Adjustable Sizes and with Exchangeable Surfactants. Chem. Mater. 2004, 16, 2509-2511.

28. Slowing, l.; Trewyn, B. G.; Lin, V. S.-Y. Effect of Surface Functionalization of MCM-41-Type Mesoporous Silica Nanoparticles on the Endocytosis by Human Cancer Cells. J. Am. Chem. Soc. 2006, 128, 14792-14793.
29. Lu, J.; Liong, M.; Zink, J. I.; Tamanoi, F. Mesoporous Silica Nanoparticles as a Delivery System for Hydrophobic Anticancer Drugs. Small 2007, 3, 1341-1346.

30. Yi, D. K.; Lee, S. S.; Papaefthymiou, G. C.; Ying, J. Y. Nanoparticle Architectures Templated by $\mathrm{SiO}_{2}-\mathrm{Fe}_{2} \mathrm{O}_{3}$ Nanocomposites. Chem. Mater. 2006, 18, 614-619.

31. Lang, N.; Tuel, A. A Fast and Efficient lon-Exchange Procedure To Remove Surfactant Molecules from MCM-41 Materials. Chem. Mater. 2004, 16, 1961-1966.

32. Grün, M.; Lauer, I.; Unger, K. K. The Synthesis of Micrometerand Submicrometer-Size Spheres of Ordered Mesoporous Oxide MCM-41. Adv. Mater. 1997, 9, 254-257.

33. Xu, H.; Yan, F.; Monson, E. E.; Kopelman, R. RoomTemperature Preparation and Characterization of Poly(ethylene glycol)-Coated Silica Nanoparticles for Biomedical Applications. J. Biomed. Mater. Res., Part A 2003 66A, 870-879.

34. Zhang, Z.; Berns, A. E.; Willbold, S.; Buitenhuis, J. Synthesis of Poly(ethylene glycol) (PEG)-Grafted Colloidal Silica Particles with Improved Stability in Aqueous Solvents. J. Colloid Interface Sci. 2007, 310, 446-455.

35. Gerion, D.; Pinaud, F.; Williams, S. C.; Parak, W. J.; Zanchet, D.; Weiss, S.; Alivisatos, A. P. Synthesis and Properties of Biocompatible Water-Soluble Silica-Coated CdSe/ZnS Semiconductor Quantum Dots. J. Phys. Chem. B 2001, 105, 8861-8871.

36. Bagwe, R. P.; Hilliard, L. R.; Tan, W. Surface Modification of Silica Nanoparticles to Reduce Aggregation and Nonspecific Binding. Langmuir 2006, 22, 4357-4362.

37. Corot, C.; Robert, P.; Idee, J.-M.; Port, M. Recent Advances in Iron Oxide Nanocrystal Technology for Medical Imaging. Adv. Drug Delivery Rev. 2006, 58, 1471-1504.

38. Sudimack, J.; Lee, R. J. Targeted Drug Delivery via the Folate Receptor. Adv. Drug Delivery Rev. 2000, 41, 147-162.

39. Kam, N. W. S.; O'Connell, M.; Wisdom, J. A.; Dai, H. Carbon Nanotubes as Multifunctional Biological Transporters and Near-Infrared Agents for Selective Cancer Cell Destruction. Proc. Natl. Acad. Sci. U.S.A. 2005, 102, 11600-11605.

40. Mal, N. K.; Fujiwara, M.; Tanaka, Y.; Taguchi, T.; Matsukata, M. Photo-Switched Storage and Release of Guest Molecules in the Pore Void of Coumarin-Modified MCM-41. Chem. Mater. 2003, 15, 3385-3394.

41. Nguyen, T. D.; Liu, Y.; Saha, S.; Leung, K. C.-F.; Stoddart, J. F.; Zink, J. I. Design and Optimization of Molecular Nanovalves Based on Redox-Switchable Bistable Rotaxanes. J. Am. Chem. Soc. 2007, 129, 626-634

42. Zhu, Y.; Fujiwara, M. Installing Dynamic Molecular Photomechanics in Mesopores: A Multifunctional Controlled-Release Nanosystem. Angew. Chem., Int. Ed. 2007, 46, 2241-2244.

43. Lee, R. J.; Low, P. S. Delivery of Liposomes into Cultured KB Cells via Folate Receptor-Mediated Endocytosis. J. Biol. Chem. 1994, 269, 3198-3204.

44. Soppimath, K. S.; Liu, L. H.; Seow, W. Y.; Liu, S. Q.; Powell, R.; Chan, P.; Yang, Y. Y. Multifunctional Core/Shell Nanoparticles Self-Assembled from pH-Induced Thermosensitive Polymers for Targeted Intracellular Anticancer Drug Delivery. Adv. Funct. Mater. 2007, 17, 355-362. 